\title{
Developing the Transliteration Interface for Arabic Text
}

\author{
Mohammed Messaoudi \\ Imam Mohammad Ibn Saud Islamic University, College of Sciences, \\ Riyadh, Saudi Arabia
}

\begin{abstract}
:
In the Arabic-English and English-Arabic translation activities, the interface is very significant. For translation in the Arabic language, many issues need to be addressed. The existing systems have some problems and research has been initiated to improve. Transliteration is an important component of the translation. We in this study propose a system of interface for Arabic transliteration. The interface shows significance and we do hope to improve further in the forthcoming studies.
\end{abstract}

\section{General Terms}

Arabic Language, Transliteration, Arabic-English Translation, Information Retrieval.

\section{Keywords}

Arabic, Transliteration, interface, Translation

\section{INTRODUCTION}

In the last decade significant progress is achieved in the information retrieval process as information systems face the challenge of managing the overload of information. The information overload has increased with the spread of communication networks and the rise of the World Wide Web (WWW). This is largely because of the ever-increasing amounts of unstructured data they make available to users. As with English, a massive volume of textual data is available on the net in the Arabic language, but text mining tools and techniques are mostly English language oriented. Therefore an efficient text mining tool to cover both Arabic and English texts simultaneously is lacking. To resolve this limitation within text mining in a uniform fashion, we propose a framework in this paper, namely the Interface for Arabic transliteration for performing the automatic task.

The plan for this study is divided into three interface stages with graphical display to motivate the general users. In this paper we present a general framework capable of achieving the above goals, and have included the interface owing to its wide acceptance. The interface approach is very efficient in organizing very large document collections onto document maps by itself [1]. The benefits of using interfaces lie in identifying the statistical features of natural text, particularly through cluster analysis and visualization. Text mining for multilingual datasets was carried out by many including $[2,3,4]$.

The Arabic language is diglossic, which is a sign of richness and complexity. It is possible to define the relationships of the Arabic language classes in the following manner:

- MA - Mother Arabic

- SA - Standard Arabic - the medium ground where heritage and language laws negotiate.

- RA - Regional Arabic
It is also important to note that the Modern Standard Arabic derives from Classical Arabic. However, only Standard Arabic is widely studied and officially used throughout the Islamic world. Modern Standard Arabic derives from Classical Arabic. [5]

A short look at any free encyclopedia [11] gives us the basics concerning the Arabic language. Arabic (al-'arabiyyah), in terms of the number of speakers, is the largest living member of the Central Semitic language family, closely related to Hebrew and Aramaic and has its roots in a Proto-Semitic common ancestor. In ISO 639-3, modern Arabic is classified as a macro-language with 27 sub-languages.

As Arabic is being spoken and used by a large number of people and countries and also the relation between English and Arabic in terms of communication increases, there is a need for automatic and effective translation activities between these two languages.

Translation process involves the consideration of Grammar, Vocabulary, Context, Phonological, Semantic and other issues. Translation is a highly complex process and a perfect solution to the automatic translation is somewhat difficult as languages differ considerably in terms of the characteristics. Automatic translation is yet to be implemented totally with success despite the huge volume of work carried out in this direction. The major breakthrough in this direction is the 'Google' language tool that enables the translation of a text from one to other language [14]. Many existing automatic translation systems currently have been developed using a rule-based approach. It involves a large volume of work based on using glossaries, vocabularies and grammars. Dictionaries are available online as open source like Qanoose

\section{Translation and Transliteration}

Full text translation or translations of words and phrases covers slang, jargon and technical terms [7]. At a recent web cast of the Google Factory Tour, researcher Franz Och presented the current state of the Google Machine Translation Systems. He compared translations of the current Google translator, and the status quo of the Google Research Lab's activities. The results were highly impressive. A sentence in Arabic which is now being translated to a nonsensical "Alpine white new presence tape registered for coffee confirms Laden" is now in the Research Labs being translated to "The White House Confirmed the Existence of a New Bin Laden Tape." [8].

It's certainly complex to program such a system, but the underlying principle is easy - so easy in fact that the researchers working on this enabled the system to translate from Chinese to English without any researcher being able to speak Chinese [10]. To the translation system, any language is treated the same, and there is no manually created rule-set of grammar, metaphors and such. Instead, the system is learning from existing human translations. Google relies on a large corpus of texts which are available in multiple languages. 
A consecutive sequence of Arabic letters, except for the punctuation marks, was recognized as a word. In an experiment by Chen and Gey [9], two online English-Arabic bilingual dictionaries and one online machine translation system were utilized in translating the English topics into Arabic in the cross-language retrieval experiments. The first online EnglishArabic dictionary is publicly accessible in the web. The English-Arabic machine translation system is also available in the web. The second one is the Ectaco dictionary publicly available at [18].

\section{Transliteration for English to Arabic}

The transliteration as a part of translation is also complex and achieving a perfect transliteration is difficult. Identification of the corresponding other language words for each of the spelling is simple for a few languages and difficult for some languages like English. A transliteration lexicon composed of many transliterated term pairs is an important resource to researches on machine transliteration. However, it is time and labor-consuming to prepare such a lexicon.

Any transliteration system has to make a number of decisions which are dependent on its intended field of application. One basic problem is that written Arabic is normally unvocalized, i.e., many of the vowels are not written out, and must be supplied by a reader familiar with the language. Hence unvocalized Arabic writing does not give a reader unfamiliar with the language sufficient information for accurate pronunciation. An exact equivalent of قطر would be qțr, which is meaningless to an untrained reader. A "full transliteration" adds information not in the text, which has to be supplied by a speaker of Arabic, qațar. Usually, newspapers and popular books do not use a transliteration, but a transcription: Instead of transliterating each written letter, they try to reproduce the sound of the words according to the orthography rules of the target language (Qatar). Transliterated-term extraction using parallel corpora has been conducted in large scale by many researchers such as [12].

Google ta3reeb (Arabic transliteration) [14] offers an option for converting Roman characters to Arabic characters. This lets you type Arabic words phonetically in English script and still have them appear correctly in Arabic. Note that this is not the same as translation -- it is the sound of the words that are converted from one alphabet to the other, not their meaning. For example, typing "ta3reeb" transliterates into Arabic as: "Because of the nature of this system, there is no single "correct" way to write an Arabic character (e.g., "ج" might be transliterated as "j" or "g"). Normally, Arabic letters are represented by a letter (or a combination of letters) that are phonetically equivalent (or nearly equivalent) in English.

Microsoft Maren [13] is a Windows extension that comes to the rescue, allowing you to type Arabic in Roman characters (Romanized Arabic, Arabizi, Arabish or Franco-Arabic) and have it converted on the fly to Arabic script. Google has now integrated Arabic Transliteration in Gmail. Also, they have a simple bookmarklet for Arabic Transliteration on any page.

The google transliteration assistant has achieved significant progress. It allows the keying of Arabic words (not letter by letter) which offers the corresponding Arabic words for transliteration. It is not vice versa like Arabic to English. Yamli [15] is similar to google transliteration interface. Web based transliteration for Arabic are proposed by many such as [16] but not implemented and there is little progress. A few projects aim to have a full translation for the interface by translating messages in certain files to Arabic. This is done by translating strings used for example in the KDE interface. QaMoose [6] facilitates the access to an English/Arabic user-defined dictionary via web access using the DICT protocol. Its inception was meant to establish and retain consistent content to better assist Arabeyes' translators and technical writers (the dictionary content is envisioned to come from Arabeyes.org' Wordlist project [17]).

\section{Discussions}

We have tested the existing transliteration interfaces such as Microsoft Maren, Google ta3reeb and Yamli. These are similar in their approach as the keying of English words produce equivalent English words but after entering the complete word or proceeding to the 'enter' after keying. They use a free text box for keying. However. we have proposed a virtual key board for keying where the end users feel that they are working practically when they feed terms for transliteration.

The interface has been developed as follows.

The interface basically has the following features.

Interface 1

Translation options

Г

Transliteration of abbreviations

Translation Theme:

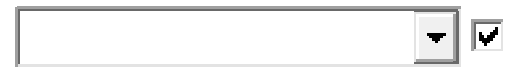

Transliteration of Proper Nouns

\section{Г} organizations etc)

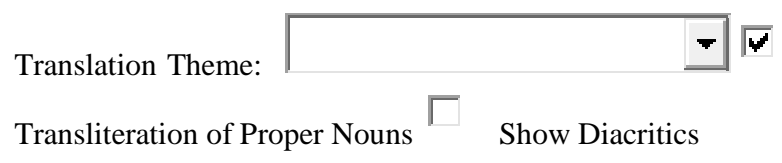

In the transliteration process the users can select the two options names (nouns) or abbreviations.

The existing systems do not use any keyboard in the interface even it is proposed like in QaMoose. We have employed the virtual keyboard in our process

Interface 2

Virtual Keyboard

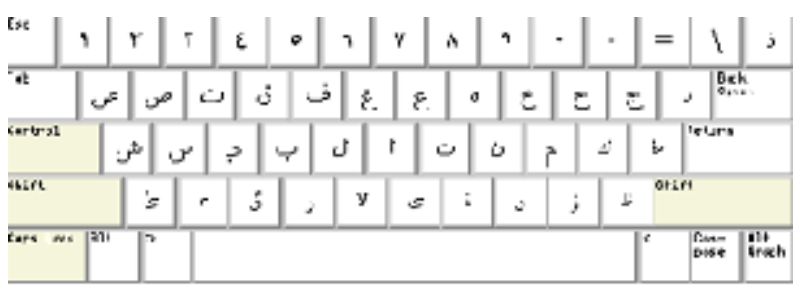

\section{Transliteration for Abbreviations}

For English Abbreviations of terms such as CD, WWW, etc the terms can be given in the following boxes. This will allow the users to interact with the systems and can suggest the transliteration. The problem in such transliteration is that the perfect transliteration will not reflect the true meaning behind such abbreviations. However we can try to achieve improved results using an user suggestive system. 
Interface 3

Top of Form

\begin{tabular}{l}
\hline add_term \\
English Abbreviation: \\
Arabic term (UTF-8) \\
(suggestive) \\
Note:
\end{tabular}

Reset Submit

In addition to the transliteration we tried to offer a few improvements in translation as follows: Google translation system by randomly feeding of large texts that consists of sentences. We feed the random selection of sentences in English and get the Arabic texts translated. The translation is found to be good, but not completely successful. It has a few difficulties. First, is the use of grammar of languages. Basically grammar of languages differs with each other. In EnglishArabic translation we found two principal difficulties. The order of the components of Arabic language translated sentences is displaced resulting the difficulties in meaning. Second is the interpretation of words that have more than one meaning.

\section{SUMMARY}

The author has documented two principal problems in the construction of interfaces in automatic transliteration and translation.

The transliteration proceeds by letter by letter and after the end of the completion of a noun or abbreviation. Besides we employed the virtual key board which is user friendly. The use of virtual keyboard will lead to the better degree of use by users.

The use of grammar and its automatic deployment is difficult and resulted in conveying proper meaning. Then second is the failure of context specification in translated version. We now intend to build a glossary of selected words in English as well as Arabic. We started to build a context glossary where one word implies many meanings.

\section{CONCLUSION}

At this stage we do not have any difficulty in the deployment of words or building vocabularies that specify the context. We presently use the existing English as well as Arabic vocabularies. The work uses the letters both in Arabic and English.

Abbreviations and Acronyms

There are many acronyms and abbreviations in many subjects and areas that include technical terminology, which makes it difficult to introduce Arabic equivalents. To resolve this issue the users are expected to do the following:

- Product names should always be transliterated in Arabic characters. This is regardless of whether they are an acronym or an abbreviation.

- All other acronyms (e.g. HTML, SGML, HTTP) should be transcribed in their Latin form (using English characters), but their translated antonyms could be added between parenthesis for clarification.

\section{FUTURE}

It is planned to improve further by developing interface for acronyms besides enhancing the present work.

\section{ACKNOWLEGMENT}

The authors are thankful to the Al Imam Muhammad bin Saud Islamic University, Riyadh, Saudi Arabia for supporting and funding this work

\section{REFERENCES}

[1] Bingham, E, Kuusisto, J., and Lagus, K. (2002). ICA and SOM in text document analysis. SIGIR02, ACM 1581135610/02/0008. Tampere, Finland..

[2] Abdulsamad Al-Marghilani, Hussein Zedan and Aladdin Ayesh (2008). Text Mining Based on Self-Organizing Map Method for Arabic-English Documents, Association for the Advancement of Artificial Intelligence, In: www.aaai.org

[3] Al-Sulaiti, L. (2006). International Corpus of Arabic (ICA). School of Computing at the University of Leeds. http://www.comp.leeds.ac.uk/eric/latifa/research.htm

[4] Silberztein, M. (2005). Nooj: a Linguistic Annotation System for Corpus Processing. In: Proc. Association for Computational Linguistics of HLT/EMNLP, Vancouver, Canada pp. 10-11. http://www.aclweb.org/anthology/H/H05/H05-2006.

[5] Fouzi Harrag, Aboubekeur Hamdi-Cherif, Abdul Malik S.Al-Salman, Eyas El-Qawasmeh (2009) 93rd International Conference on Arabic Language Processing (CITALA'09), May 4-5, 2009, Rabat, Morocco Experiments in Improvement of Arabic Information Retrieval, p. 71)

[6] The Arabic Unix Project: QaMoose, the English to Arabic http://qamoose.arabeyes.org/dict_main.cgi

[7] Babylon Translation System. http://www.babylon.com

[8] Google Blogoscoped, Google Translator: The Universal Language, http://blogoscoped.com/archive/2005-05-22n83.htm.

[9] Aitao Chen, and Fredric Gey. Translation Term Weighting and Combining Translation Cross-Language Retrieval, 2008 
[10] Jin-Shea Kuo, Ying-Kuei Yang (2008). Incorporating Pronunciation Variation into Extraction of Transliteratedterm Pairs from Web Corpora, Journal of Chinese Language and Computing 15 (1) p. 33-44.

[11] Wikipedia. Romanization of Arabic, http://en.wikipedia.org/wiki/Romanization_of_Arabic\#Tr ansliteration_standards.

[12] Lee, C. J. and Chang, J. S. (2003). Acquisition of English-Chinese Transliterated Word Pairs from ParallelAligned Texts Using a Statistical Machine Transliteration Model, In Proceedings of HLT-NAACL, Edmonton, Canada, pp. 96-103.

[13] Microsoft Afkar. Microsoft Maren Transliteration, http://www.microsoft.com/middleeast/egypt/cmic/maren
[14] Google Transliteration interface, http://www.google.com/transliterate/arabic/.

[15] Yamli Arabic Keyboard, http://www.yamli.com/arabickeyboard.

[16] Web-based Arabic Transliteration. http://neugierig.org/software/arabic/

[17] Mohammed Elzubeir, Arabic Documentation Standards. Arabeyes Project. Revision: 1.4 available at www.arabeyes.org/download/documents/misc/doc_stand ards-en.

[18] The Ectaco Online Dictionary, http://www.gettogether.net. 